\title{
Optimal cytoreduction is the only independent prognostic factor for survival in women with ovarian clear cell carcinoma
}

\author{
Ulaş Solmaz $^{1 *}$, Emre Mat ${ }^{1}$, Atalay Ekin ${ }^{1}$, Levent Dereli ${ }^{2}$, Ozgur Deniz Turan $^{3}$, Gulsah Selvi \\ Demirtas $^{1}$, Cenk Gezer ${ }^{1}$, Pinar Solmaz Hasdemir ${ }^{1}$, Sevil Sayhan ${ }^{4}$, Muzaffer Sanci ${ }^{1}$
}

\begin{abstract}
Objective: To evaluate the clinicopathological characteristics, treatment methods, survival, and prognosis of ovarian clear-cell carcinoma (OCCC).

Material and Methods: All patients with OCCC who were treated between January 1998 and October 2012 were retrospectively reviewed. After the exclusion criteria, a total of 39 women were included in the present study. Univariate and multivariate analyses were used to identify the risk factors for overall survival (OS) and progression-free survival (PFS).

Results: The majority of the patients were at stage I disease $(n=21$ [24.3\%]). All patients underwent total abdominal hysterectomy and bilateral salpingo-oophorectomy. Additionally only pelvic, and pelvic plus paraaortic lymphadenectomy was done in $8(20.5 \%)$ and $19(48.8 \%)$ women, respectively. Optimal cytoreductive surgery was achieved in $26(66.7 \%)$ patients. Recurrences occurred in $11(28.2 \%)$ patients. The median followup period was 51 months (range $4-132$ months). The 5-year PFS and OS rates were 47\% and 54\%, for all patients. The 5-year OS rates for women with early (stage I and II) and advanced (stage III and IV) stage disease were $56.4 \%$ and $38.1 \%$, respectively. Multivariate analysis confirmed optimal cytoreduction as the only independent predictor of OS [Odds ratio (OR) 21.212, 95\% confidence interval (CI) 5.259-85.556, (p<0.001)] Conclusion: Optimal cytoreductive surgery is the only independent good prognostic factor for survival in patients with OCCC.
\end{abstract}

Keywords: ovarian clear-cell carcinoma, survival, optimal cytoreduction, chemotherapy

\section{Introduction}

Ovarian cancer is the most lethal malignancy of the genital tract (1). Epithelial ovarian cancer (EOC) accounts for 90-95\% all ovarian cancer types. Ovarian clear cell carcinoma (OCCC) is a rare subtype of EOC, constituting approximately $5 \%$ to $25 \%$ of cases (2,3). These tumors were first described and originally named as 'mesonephroma ovarii' due to the patological findings including hobnailed clear-cells with an immature glomerular pattern (Fig 1) (4).

Unlike other subtypes, OCCC is more likely to be diagnosed at an earlier stage and occur unilaterally (5-7). They are generally associated with poor prognosis and distinct clinical features compared to other subtypes of EOC (5). An association between endometriosis and OCCC was described and nulliparous women are considered to be at higher risk like women with most subtypes of $\operatorname{EOC}(6,8,9)$.
The traditional management approach for OCCC is comprehensive surgical staging (surgical treatment consisted of hysterectomy, removal of the adnexae, and/or lymphadenectomy [pelvic and/or para-aortic], infracolic omentectomy or omental sampling, and/or tumor cytoreduction, if needed), followed by chemotherapy (CT).

However, no standard treatment method exists particularly in early-stage diseases (3). Besides, type of surgery and adjuvant treatment methods can vary from author to author depending on the experience and patient characteristics.

In the current study, we analysed the clinicopathological characteristics, treatment methods, survival, and prognosis of 39 women with OCCC

Received: 07-08-2015, Accepted 06-09-2015, Available Online 01-10-2015

1 Department of Gynecologic Oncology, Tepecik Education and Research Hospital, Izmir, Turkey.

2 Department of Obstetrics and Gynecology, Tavas State Hospital, Denizli, Turkey.

3 Department of Obstetrics and Gynecology, Ege University, Izmir, Turkey.

4 Department of Pathology, Tepecik Education and Research Hospital, Izmir, Turkey.

*Corresponding Author: Ulas Solmaz E-mail: drulassolmaz@gmail.com 


\section{Materials and Methods}

\section{Patients}

A retrospective review was conducted for all patients who had undergone surgery for OCCC between January 1998 and October 2012. This study was performed in accordance with the ethical standards of the Declaration of Helsinki and was approved by the local ethics committee of our institution. Patients who did not undergo surgery and patients with missing data were excluded. Besides, women with mixed-type OCCC or another primary cancer were not included in the study.

\section{Data collection}

Demographic data, such as age at diagnosis, clinical stage, parity, menopausal status, surgical and neo-adjuvant and/or adjuvant treatment details, perioperative and postoperative complications, followup data, and laboratory findings such as serum cancer antigen 125 (CA 125) levels were obtained from medical records. Histopathological findings, including, cytological analysis, primary tumor diameter (PTD), existence or non-existence of ovarian capsule rupture, pelvic $(\mathrm{P})$ and/or para-aortic (PA) lymph node involvement, and the size and location of extra-uterine metastatic tumors were retrieved from surgical pathology and cytology reports. All of the pathology slides were reviewed by an experienced gynaecologic pathologist.

\section{Surgical technique}

All of the patients underwent laparotomy. Total abdominal histerectomy (TAH) with bilateral salpingo-oopherectomy (BSO), and cytolopathological analysis of ascitic fluid were performed in all cases. Infragastric omentectomy was performed in most cases whereas resection of peritoneal implants by stripping the pelvic, abdominal, and/or diaphragmatic peritoneum was performed in some eligible cases. The decision to perform systematic $\mathrm{P}$ and PA lymphadenectomy was determined by the surgical team. No lymph nodes were sampled in some patients, only the $\mathrm{P}$ or PA nodes were sampled in some patients, bilateral P lymph node dissection (LND) was applied in some patients, and some patients underwent bilateral $\mathrm{P}$ and PA LND. Colorectal, small bowel, and upper abdominal organ resections were also performed when necessary. The general goal was to remove as much of the tumor as possible to achieve optimal cytoreduction, which was defined as residual disease $\leqslant 1 \mathrm{~cm}$ according to the Gynecologic Oncology Group (GOG). Staging criteria were determined postoperatively based on the 2009 International Federation of Gynaecology and Obstetrics (FIGO) staging system.

\section{Neo-adjuvant and adjuvant treatment}

The CT regimens were as follows: Patients were administered 3 courses of paclitaxel/carboplatin or docetaxel/carboplatin as neo-adjuvant chemotherapy (NAC) regimens. Paclitaxel was administered at a dose of $175 \mathrm{mg} / \mathrm{m} 2$ in association with carboplatin at an area under the curve of 5 or 6 (AUC 5 or 6). Docetaxel was administered at a dose of $75 \mathrm{mg} / \mathrm{m} 2$ in association with carboplatin (AUC 5 or 6). Courses were repeated every 3 weeks. Four patients underwent debulking surgery following neoadjuvant chemotherapy (NAC). To complete the full treatment regimen of 6 cycles, women in the NAC group received 3 cycles postoperatively. The patients who did not undergo NAC, received 6 cycles of CT as adjuvant $\mathrm{CT}$. The reported reasons for primary therapy with NAC were extra-abdominal disease verified by imaging methods and extensive intra-abdominal disease that was deemed unresectable by the primary surgical team. In addition, NAC was administered when the patients could not tolerate radical surgery due to advanced age, poor general condition, and/or the presence of comorbidities. Adjuvant therapy was administered to patients based on stage, age, nodal metastasis status, performance status, and the presence/absence of medical comorbidities.

\section{Clinical follow-up}

The patients returned for follow-up evaluations every 3 months for the first 2 years, every 6 months for the next 3 years, and annually thereafter. Follow-up evaluations consisted of physical and vaginal examinations, vaginal cytology, ultrasound scanning and assessment of serum CA 125 values. Computed tomography or magnetic resonance imaging was performed annually. Progression-free survival (PFS) was defined as the time from the date of primary surgery to the detection of recurrence or the latest observation. Overall survival (OS) was defined as the time interval from the date of surgery to death or last contact.

\section{Statistical analysis}

Statistical analyses were performed using IBM SPSS Statistics 22.0 (SPSS Inc., Chicago, IL). The variables were assessed using visual (histograms, probability plots) and analytical methods to determine whether they were normally distributed. Continuous data (presented as the mean $\pm \mathrm{SD}$ and median [min$\max ]$ ) were analysed using the Mann-Whitney U test for non-normal data. The chi-square test (Pearson's chi-square and Pearson's exact chi-square tests) was used to compare the proportions between groups. Univariate and multivariate logistic regression models were used to identify the risk factors. The KaplanMeier method was used to generate the survival curve, and comparisons were performed with the log rank test. A p-value $<0.05$ was defined as statistically significant. 
Results

A total of 39 patients with OCCC fulfilling the inclusion criteria were included in the present study. The median age at diagnosis was 54 years (range, 34-72 years), and 32 (82\%) women were postmenopausal. Abdominal bloating and pain $(89.7 \%)$ were the most common presenting complaints. Twenty-one patients $(53.8 \%)$ presented with FIGO stage I disease, $3(7.7 \%)$ with stage II disease, and $15(38.5 \%)$ with stage III disease. Besides, 24 (61.5\%) patients were categorised as early stage (stage I and II) and $15(38.5 \%)$ patients were categorised as advanced stage (stage III and IV). The median serum CA-125 lev $\neg$ el was $269 \mathrm{U} / \mathrm{mL}$ (range 74031). Majority of the patients $(89.7 \%)$ had serum CA-125 lev $\neg$ els $\geqslant 35 \mathrm{U} / \mathrm{ml}$. All patients had ascites at laparotomy; 26 (66.7\%) had $<500 \mathrm{cc}$ and $13(33.3 \%)$ had $\geqslant 500 \mathrm{cc}$. The demographic findings and clinicopathological characteristics are summarised in table 1.

Among 39 patients, $12(30.8 \%)$ underwent $\mathrm{TAH}+\mathrm{BSO}$. Omentectomy were performed in all women except in 3 who had undergone only TAH+BSO. $8(20.5 \%)$ underwent TAH+BSO and P lymphadenectomy, 19 (48.8\%) underwent TAH+BSO and $\mathrm{P}$ plus PA lymphadenectomy. Optimal cytoreductive surgery was achieved in 26 (66.7\%) patients. Adjuvant treatment was administered to 35 patients whereas 4 women $(13.2 \%)$ received NAC alone who had stage IIIC disease. The median PTD was $8 \mathrm{~cm}$. (range, $3-24 \mathrm{~cm}$ ). PTD were $\leqslant 8 \mathrm{~cm}$ in 11 $(28.2 \%)$ patients and $>8 \mathrm{~cm}$ in $28(71.8 \%)$.

In the present study, the univariate analysis pointed out that early stage disease and optimal cytoreduction were the significant prognostic factors for both PFS ( $p=0.021$, and $p<0.001$, respectively) and OS ( $p=0.007$ and $p<0.001$, respectively). Multivariate analysis confirmed optimal cytoreduction to be the only independent predictor of OS [Odds ratio (OR) 21.21, 95\% confidence interval (CI) 5.25-85.55, $(\mathrm{p}<0.001)]$ (Table 2) (Fig 2). No independent factors shown to affect PFS.

The median follow-up period was 51 months (range $4-132$ months). The 5-year PFS and OS rates for all patients were $47 \%$ and $54 \%$, respectively. The 5 -year OS rates for women with stage I, II, and III disease were $57 \%, 50 \%$, and $38.1 \%$ (Fig 3). On the other hand the 5-year OS rates for early and advanced stage were $56.4 \%$ and $38.1 \%$, respectively. Recurrences developed in $11(28.2 \%)$ patients, of whom 3 had stage I; 1 had stage II, and 7 had stage III disease. There was only one vaginal cuff recurrence. The rest of the recurrences were outside the $\mathrm{P}$ cavity (only PA recurrence in 5, peritonitis carcinomatosa in 3 , liver recurrence in 1 , and $\mathrm{PA}$, supraclavicular and inguinal recurrences in 1 patient).
Discussion

Ovarian clear cell carcinomas are rare tumors that have poorer outcomes and considered as one of the most aggressive ovarian tumor for they are potentially resistant to traditional platinum-based CT $(5,10)$. In the present study, the median age at diagnosis (54 years) was similar to that reported in many previous studies (11-13). Besides, $15.3 \%$ of patients were nulliparous however nulliparity has been reported to account for more than $50 \%$ of all cases in majority of the studies with the exception of the two reports $(17 \%$ and $45 \%$, respectively) $(12,13)$. The reported incidence of endometriosis in patients with OCCC ranges from $8 \%$ to $55 \%$ (14-16). In line with the literature $(8-55 \%)$, endometriosis has been reported in $20.5 \%$ of patients in our study. The percentage of OCCC patients who presented at stages I and II disease was $61.5 \%$ which has been reported to be significantly higher (53\% to $66 \%$ of patients with OCCC) compared to other subtypes of EOC (6$8,14,17-19)$. Five-year survival rates in stages I and II OCCC vary from $50 \%$ to $73 \%$ in the reported series. In our study, the 5-year OS rates for women with stage I, and II disease were $57 \%$, and $50 \%$ respectively.

Table 1. Clinical, surgical and histopathological characteristics of the study population $(n=39)$

\begin{tabular}{lc} 
Characteristic & n (\%) \\
Age (years) & $54[34-72]$ \\
Menopause & $32(82)$ \\
Yes & $7(28)$ \\
No & \\
FIGO stage & $21(53.8)$ \\
I & $3(7.7)$ \\
II & $15(38.5)$ \\
III & $269[7-4031]$ \\
CA-125 U/mL & \\
PTD & $11(28.2)$ \\
$\leq 8$ cm & $28(71.8)$ \\
$>8$ cm & $11(15.4)$ \\
Amount of ascitic fluid & $26(66.7)$ \\
$\leq 500 \mathrm{~mL}$ & $13(33.3)$ \\
$>500 \mathrm{~mL}$ & \\
Optimal cytoreductive surgery & $26(66.7)$ \\
Yes & $13(33.3)$ \\
No & \\
Recurrence & $11(28.2)$ \\
Yes & $28(71.8)$ \\
No & $24[1-132]$ \\
PFS & $35[4-132]$ \\
OS & Fedetion \\
\hline Abbreviations & FIGO, Inter \\
\hline
\end{tabular}

Abbreviations: FIGO, International Federation of Gynecology and Obstetrics; PTD, primary tumor diameter; OS, overall survival; PFS, progression-free survival. Values for the continuous variables are median (min-max). Values for the categorical variables are the number/total number of cases $(\%)$. 
Table 2. Univariate and multivariate analysis of overall survival in the patients with ovarian clear cell carcinoma

\begin{tabular}{|c|c|c|c|c|}
\hline \multirow[t]{2}{*}{ Characteristic } & \multicolumn{2}{|c|}{ Univariate model } & \multicolumn{2}{|c|}{ Multivariate model } \\
\hline & & p-value & OR $(95 \%$ CI $)$ & p-value \\
\hline $\begin{array}{l}\text { Age at surgery ( } \leq 54 \text { vs. }>54 \\
\text { years) }\end{array}$ & & 0.603 & & \\
\hline \multicolumn{5}{|l|}{ PTD } \\
\hline$\leq 8 \mathrm{~cm}$ & reference category & & & \\
\hline$>8 \mathrm{~cm}$ & & 0.333 & & \\
\hline \multicolumn{5}{|l|}{ FIGO stage } \\
\hline early stage & reference category & & & \\
\hline advanced stage & & 0.021 & & \\
\hline \multicolumn{5}{|l|}{ Optimal cytoreductive surgery } \\
\hline No & reference category & & reference category & \\
\hline Yes & & $<0.001$ & $21.21(5.25-85.55)$ & $<0.001$ \\
\hline \multicolumn{5}{|l|}{ Ascites } \\
\hline$\leq 500 \mathrm{cc}$ & reference category & & & \\
\hline$>500 \mathrm{cc}$ & & 0.274 & & \\
\hline
\end{tabular}

Abbreviations: OR, odds ratio; CI, confidence interval; LVSI, lymphovascular space invasion; PTD, primary tumor diameter; MI, myometrial invasion; FIGO, International Federation of Gynecology and Obstetrics. A pvalue of $<0.05$ was considered to be statistically significant.

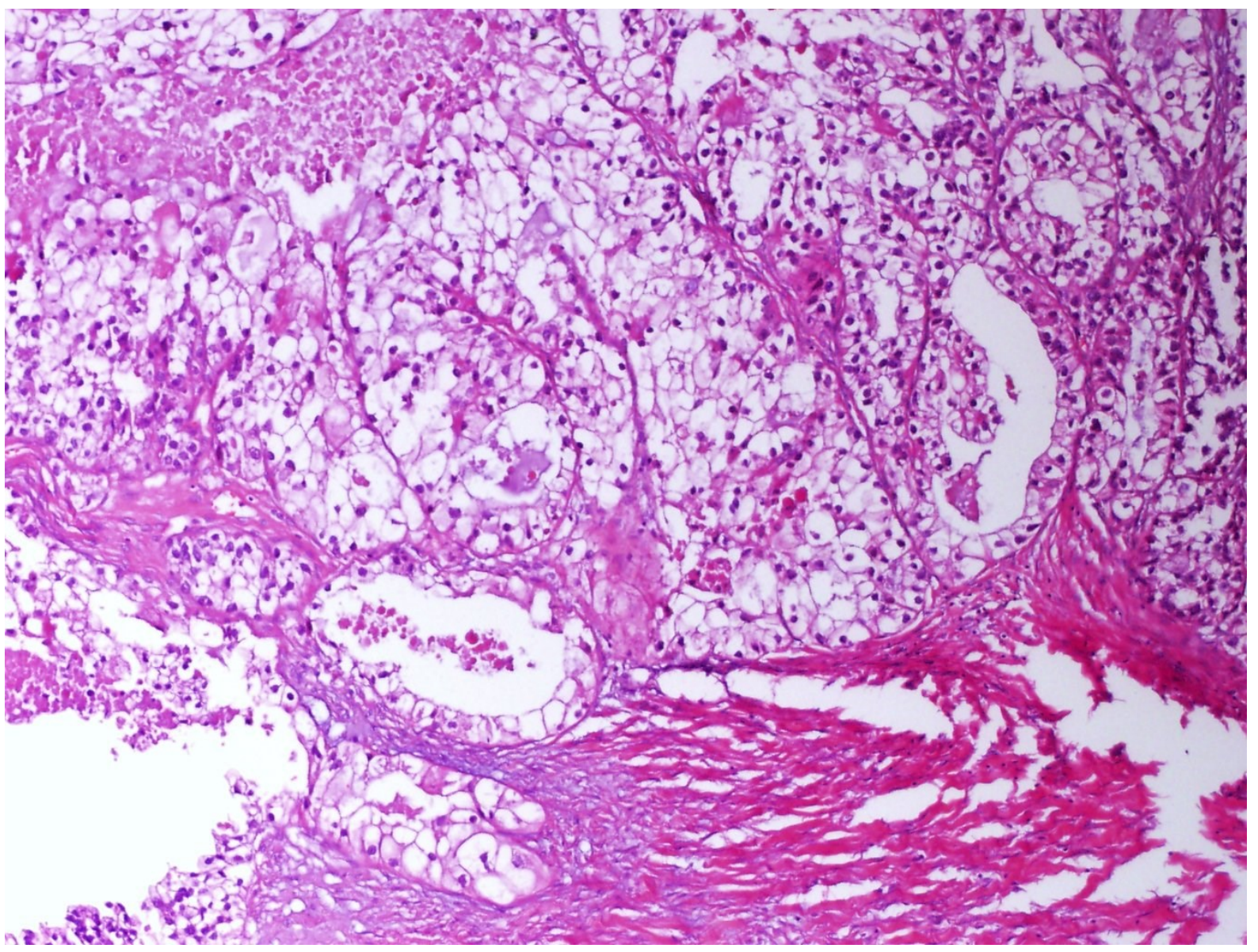

Figure 1. Clear cell of the ovary, depicting the characteristic tubulo-cystic histologic pattern $(100 \times)$ 


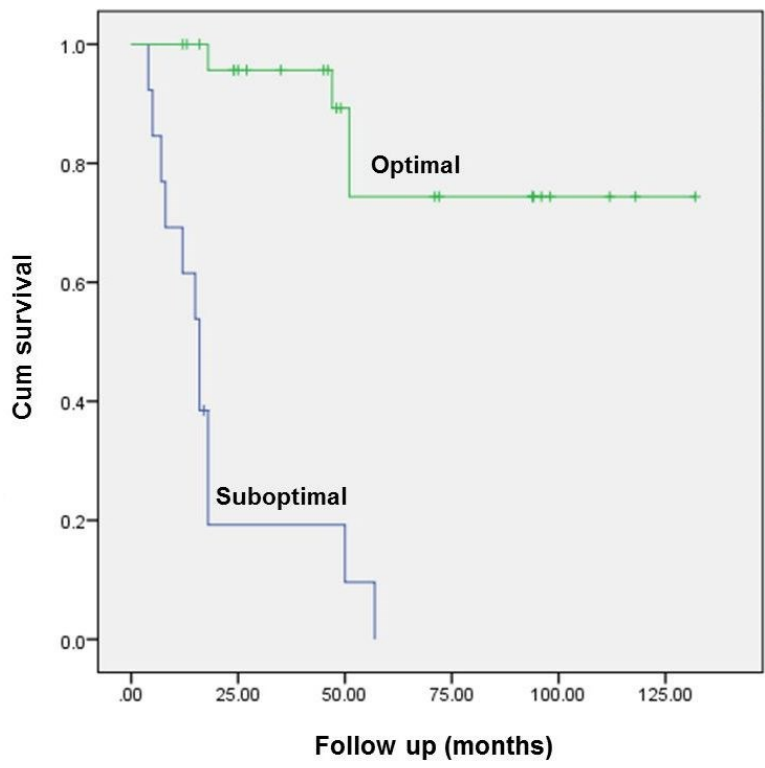

Figure 2. Kaplan-Meier curves of the clinical outcome. Overall survival of all patients $(n=39)$ when grouped according to cytoreduction (optimal vs. suboptimal)

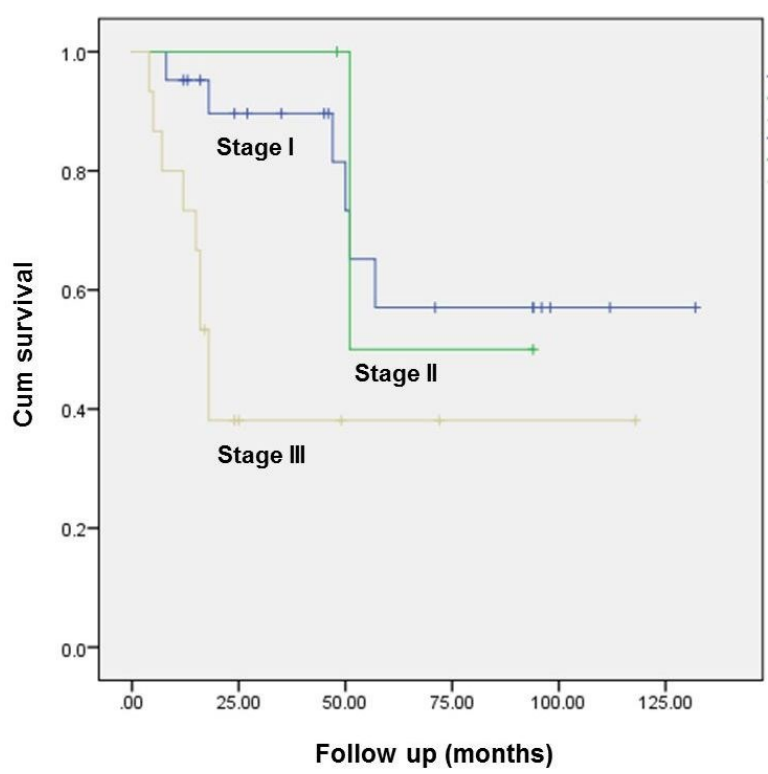

Figure 3. Kaplan-Meier curves of the clinical outcome. Overall survival of all patients $(n=39)$ when grouped according to stage

In a study by Mizuno et al. positive cytology, ascitic volume, residual tumor, and serum CA-125 level were the significant factors of survival on univariate analysis (20). CA-125 and residual tumor are popular prognostic factors that have prognostic impact in women with EOC (21-25). However, the low positivity rates of CA 125 in patients with OCCC, makes it a less useful prognostic marker in clinical practice. In the present study, the univariate analysis showed that early stage disease and optimal cytoreduction were the significant prognostic factors.
Mizuno et al. have also evaluated the patients with multivariate analysis for the first time in literature and found that early stage, $\leqslant 100 \mathrm{ml}$ ascitic volume, and no residual tumor were independent prognostic factors (20). In our study, less than $1 \mathrm{~cm}$ residual tumor was considered as optimal cytoreduction and it has been found to be the only independent favorable prognostic factor of OS.

There are some studies suggesting chemoresistant behaviour of OCCC. Behbakht et al. showed that $37 \%$ of patients with stage I OCCC who were subjected to platinum-based adjuvant CT relapsed (19). In support of this concept, in another study by Gorai et al. it was pointed out that cell-lines of OCCC have chemoresistence to cisplatin in cell culture (26). Besides, Kita et al. showed that $60 \%$ of patients with stage II disease who had macroscopic residual tumor died within 9 months after initial surgery and adjuvant cisplatin-based CT (14).

In the light of the results of previous reports and our study, optimal cytoreductive surgery and the presence of residual tumor have a strong impact on the prognosis of the patients with OCCC owing to the fact that these tumors are mostly chemoresistant. Consequently the general goal should be to remove as much of the tumor as possible to achieve no residual tumor or optimal cytoreduction, and even at an early clinical stage, patients should undergo complete staging surgery.

The limitations of this study are its retrospective nature, and some patients were treated by non-gynaecological oncologic surgeons and therefore patients were treated with different types of surgical approaches over the 15-year time period. Retrospective cohort studies are subjected to selection bias, recall bias, and unknown confounding variables, which may negatively impact the accuracy of the results. Moreover, during the 15-year study period, significant improvements in surgical techniques and adjuvant treatment may have also affected the results.

Lastly, the data did not allow definitive and comparative analyses assessing the heterogeneity of the different adjuvant therapy regimens and the information on chemoresistance was lacking. Despite these limitations, relatively a large number of patients diagnosed with this rare disease, with similar demographic characteristics were included in this study. Besides, good follow-up data were available. Additionally, the surgeries were performed at a single institution, and all pathological slides were reviewed by an experienced gynaecologic pathologist. All of these factors most likely increased the validity of the results and mitigated the limitations. 


\section{Conclusion}

Our study demonstrated that optimal cytoreduction to be the only independent prognostic factor for survival in women with OCCC. Considering the chemoresistant behaviour of OCCC, complete staging surgery and optimal cytoreduction surgery remains the primary treatment modality. Therefore, quality of life issues, operability and the most appropriate and effective treatment regimens should also be considered for management. Further improvements in survival rates require the optimization of adjuvant therapy modalities.

Acknowledgements: The authors would like to thank Ece Biliner for her assistance with statistical analysis.

Conflict of interest statement: Authors declare that there is no financial support or relationships that may pose potential conflict of interest.

\section{References}

1. Siegel R, Ward E, Brawley O, Jemal A. Cancer statistics, 2012. CA Cancer J Clin 2012;62:10-29.

2. Anglesio MS, Carey MS, Köbel M, Mackay H, Huntsman DG; Vancouver Ovarian Clear Cell Symposium Speakers. Clear cell carcinoma of the ovary: a report from the first Ovarian Clear Cell Symposium, June 24th, 2010. Gynecol Oncol. 2011;121:407-15

3. del Carmen MG, Birrer M, Schorge JO. Clear cell carcinoma of the ovary: a review of the literature. Gynecol Oncol. 2012;126:481-90.

4. Schiller W. Mesonephroma ovarii. Am J Cancer 1939;35:1-21

5. Goff BA, Sainz de la Cuesta R, Muntz HG, Fleischhacker D, Ek M, Rice LW,et al. Clear cell carcinoma of the ovary: a distinct histologic type with poor prognosis and resistance to platinum-based chemotherapy in stage III disease. Gynecol Oncol. 1996;60:412-7

6. Kennedy A W, Biscotti C V, Hart W R, Webster D. Ovarian clear cell adenocarcinoma. Gynecol Oncol. 1989;32:342-9.

7. Czernobilsky B, Silverman BB, Enterline HT. Clear-cell carcinoma of the ovary. A clinicopathologic analysis of pure and mixed forms and comparison with endometrioid carcinoma. Cancer. 1970;25:762-72.

8. Jenison EL, Montag AG, Griffiths CT, Welch WR, Lavin PT, Greer J, et al. Clear cell adenocarcinoma of the ovary: a clinical analysis and comparison with serous carcinoma. Gynecol Oncol. 1989;32:65-71

9. Crozier MA, Copeland LJ, Silva EG, Gershenson DM, Stringer CA. Clear cell carcinoma of the ovary: a study of 59 cases. Gynecol Oncol. 1989;35:199-203.
10. Pectasides D1, Fountzilas G, Aravantinos G, Kalofonos C, Efstathiou H, Farmakis D, et al. Advanced stage clear-cell epithelial ovarian cancer: the Hellenic Cooperative Oncology Group experience. Gynecol Oncol. 2006;102:285-291.

11. Timmers PJ, Zwinderman AH, Teodorovic I, Vergote I, Trimbos JB. Clear Cell Carcinoma Compared to Serous Carcinoma in Early Ovarian Cancer Same Prognosis in a Large Randomized Trial. Int J Gynecol Cancer. 2009;19: 88-93

12. Doshi N, Tobon H. Primary clear cell carcinoma of the ovary. An analysis of 15 cases and a review of the literature. Cancer. 1977;39:2658-2664.

13. Fine G, Clarke HD, Horn RC. Mesonephroma of the ovary. A clinical, morphological, and histogenic appraisal. Cancer. 1973;31:398-410.

14. Kita T, Kikuchi Y, Kudoh K, Takano M, Goto T, Hirata J, et al. Exploratory study of effective chemotherapy to clear cell carcinoma of the ovary. Oncol Rep. 2000;7:327-331.

15. Aure JC, Hoeg K, Kolstad P. Mesonephroid tumors of the ovary: clinical and histopathologic studies. Obstet Gynecol. 1971;37:860-867

16. Russel P. The pathologic assessment of ovarian neoplasms. Introduction to the common 'epithelial' tumors and analysis of benign 'epithelial' tumors. Pathology. 1979;11:5-26.

17. Yoonessi M, Weldon D, Satchidand SK, Crickard K. Clear cell ovarian adenocarcinoma. J Surg Oncol. 1984;27:28997

18. O'Brien ME, Schofield JB, Tan S, Fryatt I, Fisher C, Wiltshaw E. Clear cell epithelial ovarian cancer (mesonephroid): bad prognosis only in early stages. Gynecol Oncol. 1993;49:250-54.

19. Behbakht K, Randall TC, Benjamin I, Morgan MA, King S, Rubin SC. Clinical characteristics of clear cell carcinoma of the ovary. Gynecol Oncol. 1998;70: 255-58.

20. Mizuno M, Kikkawa F, Shibata K, Kajiyama H, Ino K, Kawai M, et al. Long-Term Follow-Up and Prognostic Factor Analysis In Clear Cell Adenocarcinoma of The Ovary. Journal of Surgical Oncology 2006;94:138 - 143.

21. Fisken J, Leonard RC, Stewart M, Beattie GJ, Sturgeon C, Aspinall L, et al. The prognostic value of early CA125 serum assay in epithelial ovarian carcinoma. Br J Cancer $1993 ; 68: 140-145$.

22. Parker D, Bradley C, Bogle SM, Lay J, Masood M, Hancock AK, et al. Serum albumin and CA125 are powerful predictors of survival in epithelial ovarian cancer. Br J Obstet Gynaecol 1994;101:888-893. 
23. Omura GA, Brady MF, Homesley HD, Yordan E, Major FJ, Buchsbaum HJ, et al. Long-term follow up and prognostic factor analysis in advanced ovarian carcinoma: The Gynecologic Oncology Group experience. J Clin Oncol 1991;9:1138-1150.

24. Hoskins WJ, Bundy BN, Thigpen JT, Omura GA. The influence of cytoreductive surgery on recurrence-free interval and survival in small-volume stage III epithelial ovarian cancer: A Gynecologic Oncology Group study. Gynecol Oncol 1992;47:159-166.
25. Hoskins WJ, McGuire WP, Brady MF, Homesley HD, Creasman WT, Berman M, et al. The effect of diameter of largest residual disease on survival after primary cytoreductive surgery in patients with suboptimal residual epithelial ovarian carcinoma. Am J Obstet Gynecol 1994;170:974-980

26. Gorai I, Nakazawa T, Miyagi E, Hirahara F, Nagashima Y, Minaguchi H. Establishment and characterization of two human ovarian clear cell adenocarcinoma lines from metastatic lesions with different properties. Gynecol Oncol. $1995 ; 57: 33-46$

Copyright (C) 2014 The Author(s); This is an open-access article distributed under the terms of the Creative Commons Attribution License (http://creativecommons.org/licenses/by/4.0), which permits unrestricted use, distribution, and reproduction in any medium, provided the original work is properly cited. All Rights reserved by international journal of Medical Science and Discovery. 